\author{
Military Technical College \\ Kobry El-Kobbah, \\ Cairo, Egypt
}

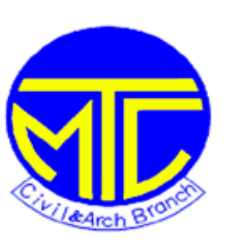

$11^{\text {th }}$

\title{
MULTI-RESOLUTION ANALYSIS ON LIDAR DATA FOR BUILDING EXTRACTION
}

\author{
Hassan Elsaid Elhifnawy \\ Civil Engineering Department, Military Technical College, Cairo, Egypt
}

KEY WORDS: LIDAR, MRA, Wavelet Transform

\begin{abstract}
:
Airborne LIDAR has become commercially used for many environmental, engineering and civil applications, and can provide accurate data for topographic surfaces and non-terrain objects. Feature extraction is one of the important applications in the field of photogrammetry. This application is used for many civil and military applications - such as photo-interpretation, vegetation, forest monitoring, traffic and transportation development and urban planning. LIDAR Data can have much more dense point spacing than is typically derived from photogrammetry and therefore proper handling of the data is needed to optimal feature extraction.
\end{abstract}

Wavelet transform techniques are widely used as powerful tools in many image processing applications such as de-noising and compression. The discrete wavelet transform is particularly suitable in de-noising and filtering problems. The properties of the wavelet transform, such as having compact support, space and frequency localization, a wide variety of base functions, denoising, thresholding, and multi-resolution analysis, are the main motivations for testing the wavelet transform as an estimation technique for feature extraction from LIDAR data.

The major objective of this research is to test the efficiency of wavelet transform in analysing LIDAR data for feature extraction applications. Wavelet transform succeeded in detecting positions of sudden changes of the geometrical or physical content of the images from LIDAR data.

\section{INTRODUCTION}

LIDAR data provides full spatial information for ground and non-ground objects, so it produces significant height information for different features. Features can be extracted by segmenting LIDAR points related to their grouping shapes or height information or both or by filtering LIDAR points to separate ground and non-ground points.

There are many algorithms for feature extraction using wavelet transform as $\mathrm{Vu}$ and Tokunaga [2001], [2002] [2004]. They produced a new clustering technique based on wavelet analysis that is called Airborne Laser Scanner Wavelet (ALSwave) for LIDAR data. The wavelet multi-resolution 
frame work is prepared based on a trous algorithm. This researchInternationhidactonfeterce wavelet transform and fuzzy edge pixels to detect object points ond eqfileathdPAinthfeegure DTM and DSM for the study area. Wang and Hsu [2006] detected building boyndaries using spatial edge detection technique and analysed edge image using wavelet transform. after applying Canny edge detection technique. The resulting image corlans ed $\mathbf{1}-\mathbf{2 0 1 6}$ all nonground points including trees and buildings. Wavelet transform is applied on the resulting image to detect finer edges related to the third level of decomposition. This edges represent building boundaries. Falkowski et al. [2006] succeeded in extracting specific type of trees, conifer tree, from LIDAR data. The first tree detection technique is the Variable Window Filter (VWF). This filter is based on detection the local maxima (tree height) through the Canopy height Model (CHM) within specific window size. It is required to have a prior knowledge about the tree height and crown diameter. This filter estimates the fixed relation between height and crown diameter, this is the major disadvantage for this technique. The second tree detection technique is Spatial Wavelet Analysis (SWA), wavelet analysis is executed using 2-D Mexican Hat wavelet function, this function is of large range from $\mathrm{m} 1$ to $15 \mathrm{~m}$ and with increments of 0.1 . Mexican Hat wavelet function is suitable for this specific tree type since its shape is similar like conifer tree shape. This filter doesn't require a prior knowledge about tree height or crown diameter. This technique explains wavelet analysis efficiency for extracting conifer trees, but it is not suitable for all trees types. Separation of ground and non-ground points in hilly terrain is not easy especially when the slope is not steep and uniform. Bartels and Wei [2006] overcome this problem using wavelet analysis and extract buildings from hilly terrain. Second order Daubechies wavelet filter is used for applying wavelet transform for the LIDAR image. The results are sub-images representing approximation and three details. The approximation represents the ground points and points for regularly distribution. The regularly distribution is formed from points with low degree of inclination or flat areas. The details represent the locations for the sharp inclination between adjacent points. The object image is obtained by reconstruction of 2D Discrete Wavelet Transform (DWT) after replacing the approximation image by zeros. At the same year they investigated a segmentation technique that can be used to separate ground and non-ground areas from LIDAR data based on wavelet analysis [Wei and Bartels, 2006]. This technique is composed of three main steps. The first step is representation of LIDAR data as gray scale image. This image is analysed by using Gabor wavelet function to three scales with four different orientations using different windows, this step is considered as the main processing step. Statistical analysis is applied for wavelet coefficients in each window. This technique succeeded in segmentation of flat (ground) areas and non- ground areas, but it has a problem in identification buildings with large flat roof as non-ground areas, then it is important to use additional classification information to able to segment all features in the input image. Parrish [2008] succeeded in detecting vertical obstruction based on 3-D wavelet representation. 3D wavelet transform function is designed based on custom requirements to serve feature extraction that has certain horizontal and vertical structures. It has many benefits like good space localization, recording sharp edge with no sensitivity to linear transition, and it has fast processing in reconstruction. This process is used for obstruction detection and it can be performed over the original 3D intensity image and for each sub-image at every level of decomposition.

These techniques used additional aiding to extract buildings as Canny edge detection [Wang and Hsu, 2006] or wavelet analysis on a trous algorithm method [ $\mathrm{Vu}$ and Tokunaga, 2002] or it is necessary to represent a LIDAR data in TIN format [Parrish, 2008].

This research paper introduces a wavelet based feature extraction technique without aiding with additional techniques or software as ENVI [Yu et al., 2005]. The proposed technique will chose wavelet base function that suitable for extracting all non-ground features not for specific feature as Mexican Hat that are suitable for specific type of trees [Falkowski et al., 2006]. 


\section{LIDAR}

A LIDAR system provides dense positioning information for surface topography and all features for the study area. LIDAR system provides not only positioning information but also property information that is called intensity values for all scanned points. Two types of images can be produced from this information, a range image and an intensity image. Range image is based on height values, while intensity image is based on intensity values for all scanned points [AlDurgham, 2007]. LIDAR system is composed of many sensors to get this information with high density and accuracy.

\subsection{LIDAR System Components}

A LIDAR system consists of two main components, a collecting information component and a navigation component. For the collecting information part, laser scanner is the main sensor as it collects both dense range and intensity information for the scanned area [Al-Durgham, 2007; ElSheimy et al., 2005; Habib et al., 2009]. Newer versions of LIDAR system contain additional collecting information sensors such as a digital camera and/or an IR sensor. These sensors collect not only positional information but also semantic information for each scanned point. On the other hand, the navigation component is composed of two systems, the Global Positioning System (GPS) and the Inertial Navigation System (INS).

\subsection{Data Acquisition}

The laser scanner contains a rotating mirror that reflects the laser beam and produce required scanning pattern. Laser scanner may contain one mirror; in this case, the scanning pattern can be linear, zigzag, or nutating. The scanner pattern takes ellipsoidal shape when laser scanner contains two rotating mirrors. Linear scanning pattern is not preferable because there is no data collected for areas between lines. Zigzag scanning pattern provides continuous collecting data for scanning area. Nutating scanning pattern provides dense collecting data with low flying speed. Elliptical scanning pattern is preferable because it gives dense collecting data with any speed since it makes duplication of collecting data for scanned area but with more complicated calculations due to existing of two mirrors that rotating in different scanning angles [El-Sheimy et al., 2005].

\subsection{Data Processing}

Inertial Measurement unit (IMU) collects navigation data for flying mission, a GPS antenna collects coordinates of flying directions referenced to the GPS coordinate system, and laser scanner collects range and intensity information for each scanned point. IMU and GPS provide integrated navigation data; they are used to calculate coordinates of the flying direction referenced to ground coordinate system. These coordinates are used with LIDAR system calibration parameters and range information to calculate ground coordinates for each point. A LIDAR system is a direct georeferencing system, so the ground coordinates of point of interest are calculated as a vector summation as shown in Figure 1 that represents a LIDAR equation [El-Sheimy et al., 2005]. Equation 1 [Habib et al., 2009] represents a mathematical model of LIDAR equation for calculating ground coordinate of point of interest. 


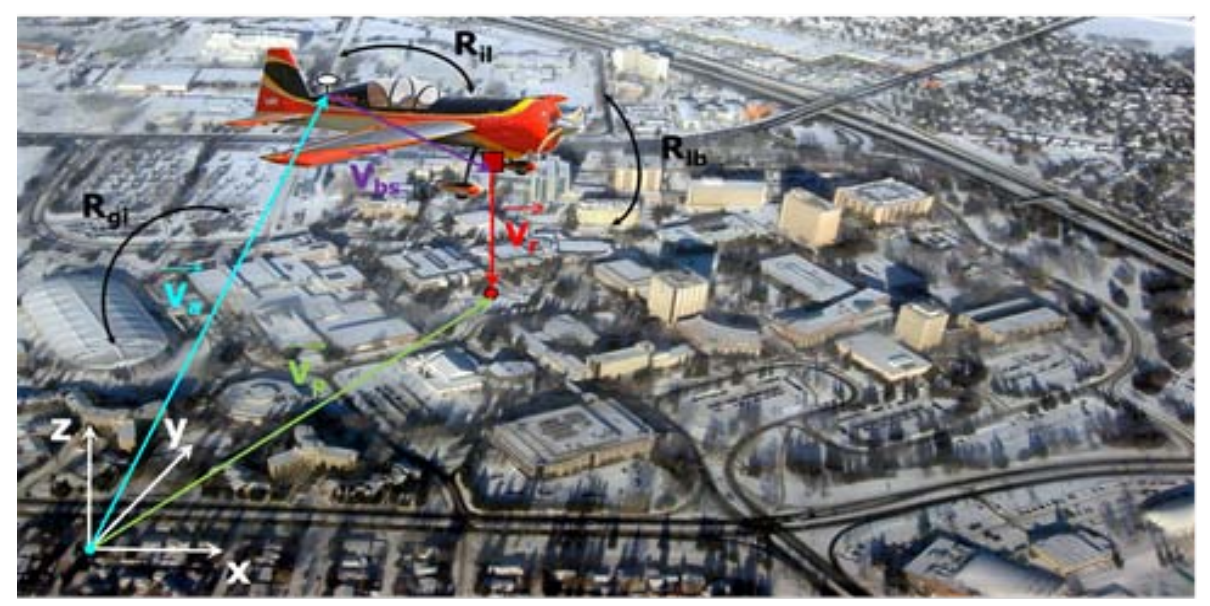

Figure 1: LIDAR Coordinate System

$$
\overrightarrow{V_{p}}=\overrightarrow{V_{\mathbb{Q}}}+R_{g i} \overrightarrow{V_{b s}}+R_{g i} R_{i \mathbb{i n}} R_{\mathbb{I b}} \overrightarrow{V_{r}}
$$

where: $\quad \overrightarrow{V_{p}}=$ Ground coordinates of point of interest

$$
\begin{aligned}
\overrightarrow{V_{a}} & =\text { Ground Coordinates of antenna phase center } \\
\overrightarrow{V_{\mathfrak{Q}}} & =\text { Vector of lever arm between antenna phase center and center of laser scanner } \\
& \text { coordinate system with respect to IMU coordinate system } \\
\vec{V}_{\boldsymbol{g}} & =\text { Vector of range of point of interest with respect to laser beam coordinate system } \\
R_{g} & =\text { Rotation matrix between IMU coordinate system and ground coordinate } \\
R_{\tilde{I}} & =\text { Rotation matrix between IMU coordinate system and laser scanner coordinate system } \\
R_{\mathbb{I}} & =\text { Rotation matrix between laser scanner coordinate and laser beam coordinate system }
\end{aligned}
$$

Positional, spatial and intensity information are gridded using an interpolation technique to produce LIDAR images, a range image and an intensity image. The grid is the same for both images but for a range image the pixel values represent the height of scanned points, and for an intensity image the pixel values represent intensity values [Al-Durgham, 2007].

\section{MULTI-RESOLUTION ANALYSIS FOR FEATURE EXTRACTION}

The proposed feature extraction technique from LIDAR data is based on Multi-Resolution Analysis (MRA) using wavelet transform. This section introduces an overview about the wavelet transform magnifying its advantages and suitability for feature extraction application.

\subsection{Wavelet Transform}

Wavelet, as a MRA method, is a powerful tool for de-noising and compressing signals and images using many thresholding techniques [Cheng et al., 2004; Jansen and Bultheel, 1999].

Wavelet has many applications with LIDAR system output; it can be applied with LIDAR signals, LIDAR data, and image investigation from LIDAR data. It can be used for de-noising LIDAR signals [Yin and Wang, 2006] and for data compression to solve storage problem of LIDAR data without significant effect on the derived surface elevation data [Pradhan et al., 2005]. Wavelet is also used for filtering and smoothing the LIDAR data for the separation between high and low frequency signal content. The filtering of the LIDAR data can be performed for the range data, raw data and commonly used for the surface elevation derived from the LIDAR data using discrete wavelet analysis [Tate et al., 2005]. 
Feature extraction application from LIDAT data is based on wavelet analysis, so before talking about feature extraction it is important to mention why wavelet is a suitable tool for this application. The following subsections explain the wavelet history and its advantages compared to Fourier transform because Fourier transform has been the basis of digital signal processing applications for many years [Elhabiby, 2007].

\subsection{One Dimensional Multi- Resolution Analysis}

In 1987 wavelet used as a basis of Multi-Resolution Analysis (MRA) that analyzes a signal or image at many resolutions. The multi-resolution analysis procedure gives the idea of wavelet filtering process. This process analyse the input signals into two main passes, high-pass filter that presents the details and the low-pass filter that presents the approximations [Gonzalez and Woods, 2002]. Multi-resolution analysis (MRA) technique is used for fast analysis of a signal into its frequency bands. This process is iterated, the resulting approximation is decomposed to its finer frequency components with dyadic down sampling, so that one signal is divided into many fine resolution components as known as Mallat algorithm as shown in Figure 2 which $\mathrm{C}$ is the indication of approximations and $\mathrm{D}$ is the indication of details. The final signal is the result of the final approximation and the summation of all details as shown in Equation 2 [Keller, 2004].

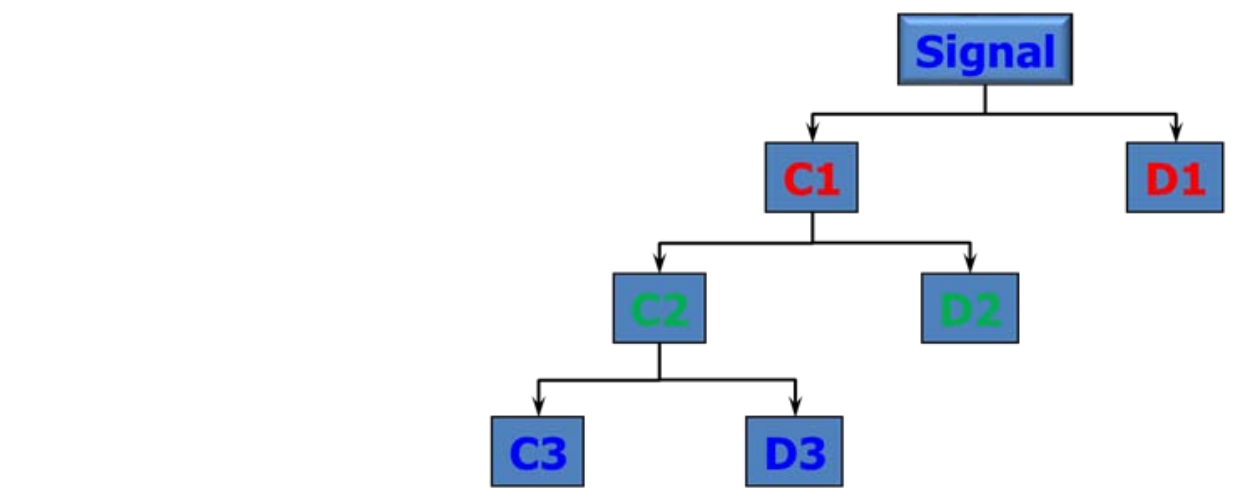

Figure 2: Multi- Resolution Analysis

$f(x)=\sum_{n} c_{\mathbb{N}}(n) \varphi_{\mathbb{N}, n}(x)+\sum_{m=1}^{N} \sum_{n \in Z} d_{m}(n) \psi_{m, n}(x) \quad 2$

where: $\quad c=$ scaling coefficient

$d$ = detail coefficient

$\mathrm{N}=$ total number of level of decompositions

\subsection{Two Dimensional Multi- Resolution Analysis}

The image is a two dimensional function for each direction $\mathrm{X}$ and $\mathrm{Y}$. There are two analysing functions phi and psi in each direction. Stefen Mallat applied the tensor product between scaling and wavelet functions in two directions to investigate the scaling function and wavelet functions (Equation 3). This process tends to get four analysing functions scaling and three wavelet functions in three directions (horizontal, vertical, and diagonal). Finally the image at each level of decomposition is analysed into one approximation and three details horizontal, vertical, and diagonal. 


$$
\begin{aligned}
& \varphi\left(x_{z} y\right)=\varphi(x) \odot \varphi(y) \\
& \psi^{H}\left(x_{z} y\right)=\psi(x) \odot \varphi(y) \\
& \psi^{V}\left(x_{v} y\right)=\varphi(x) \odot \psi(y) \\
& \psi^{D}\left(x_{v} y\right)=\psi(x) \odot \psi(y)
\end{aligned}
$$

where: $\quad \varphi\left(x_{s} y=\right.$ scaling function

$\psi^{H}\left(x_{x_{s}^{\prime}}=\right.$ horizontal wavelet function

$\psi^{V}\left(x_{i}=\right.$ vertical wavelet function

$\psi^{D}\left(x_{x_{i}}=\right.$ diagonal wavelet function

Equation 4 shows the mathematical model for scaled and translated two-dimensional scaling and wavelet functions where $\lambda_{0}$ equals to two and $t_{0}$ equals to one [Gonzalez et al., 2004; Keller, 2004].

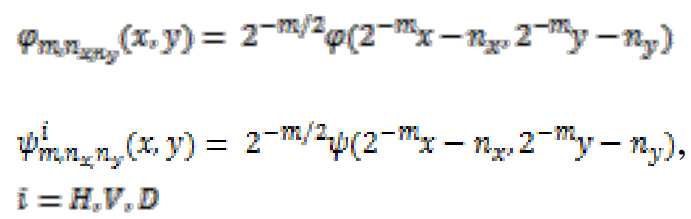

\section{LIDAR DATA FOR STUDY AREA}

Since the main target of this research is the feature extraction, then the selection of the study area was concentrated on the region with rich of buildings, this condition is verified in the selected study area that is located in Calgary, Canada and covers a part of university of Calgary campus. The study area was scanned by Optic ALTM 3100 LIDAR system.

The study area is the fourth strip from this project, it is bounded with $\mathrm{X}$ coordinates from $699915.05583963 \mathrm{~m}$ to $701414.63341027 \mathrm{~m}$ and $\mathrm{Y}$ coordinates from $5661929.8682168 \mathrm{~m}$ to $5662769.353172 \mathrm{~m}$. LIDAR system scanned this area by zigzag scanning system with total number of scanned points equals to 982956 points.

The distribution of scanned points is irregular, it is necessary to regularize this distribution to produce height and intensity images. Redistribution these points by using interpolation technique to generate equally spaced mesh and calculate height and intensity information for each node. Nearest Neighbor (NN) interpolation technique is applied to generate height and intensity images over 0.5 m equally spaced mesh. 


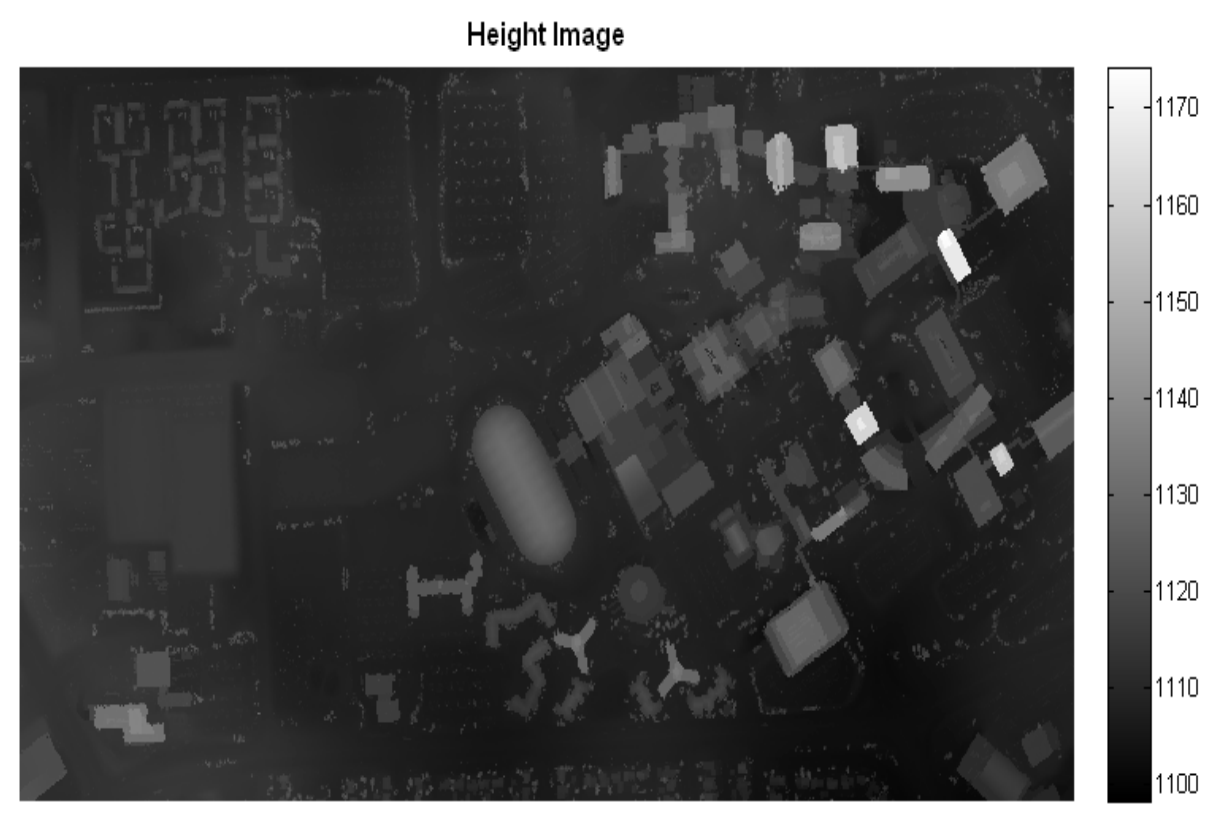

Figure 3: Height Image

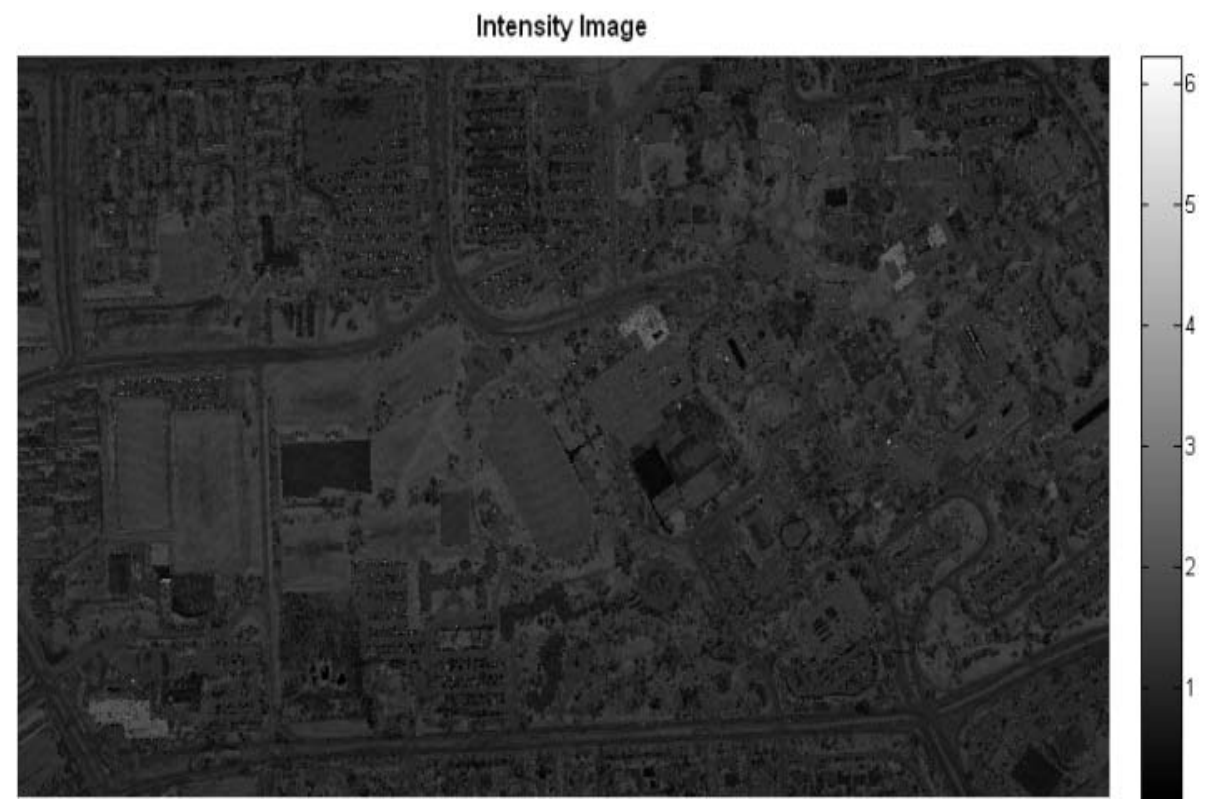

Figure 4: Intensity Image

\subsection{Wavelet Analysis}

Since there are many wavelet base functions it is difficult to identify the suitable wavelet function for the target of this research. This research tested many wavelet base functions for different levels of decomposition to get the most suitable wavelet function and optimum decomposition level for building extraction application. The main aim is the identifying the location of suddenly change in frequency that represents the building boundary. Then it is important to concentrate on the details that record the high frequencies.

After testing many different wavelet base functions, it is found the Sym4 wavelet base function (Figure 5) at the second level of decomposition gives better results for building boundary identification. The evaluation of this wavelet function is based on visual comparison between building boundary images derived from each wavelet function. The comparison was concentrating on the loss of building boundary pixels and the existence of other features. Sym 4 wavelet function gives better boundary identification with minimum existence of other features. 

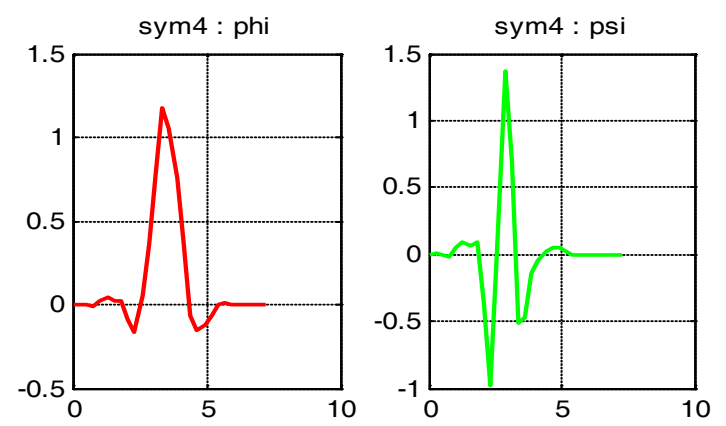

Figure 5: Sym4 Wavelet Base Function

The height image is analysed by SYM4 wavelet function to two levels of decompositions; wavelet function decomposes height image into approximation and six details as shown in Figure 6. The second step is the eliminating low frequency components which is called approximation (Figure 7).

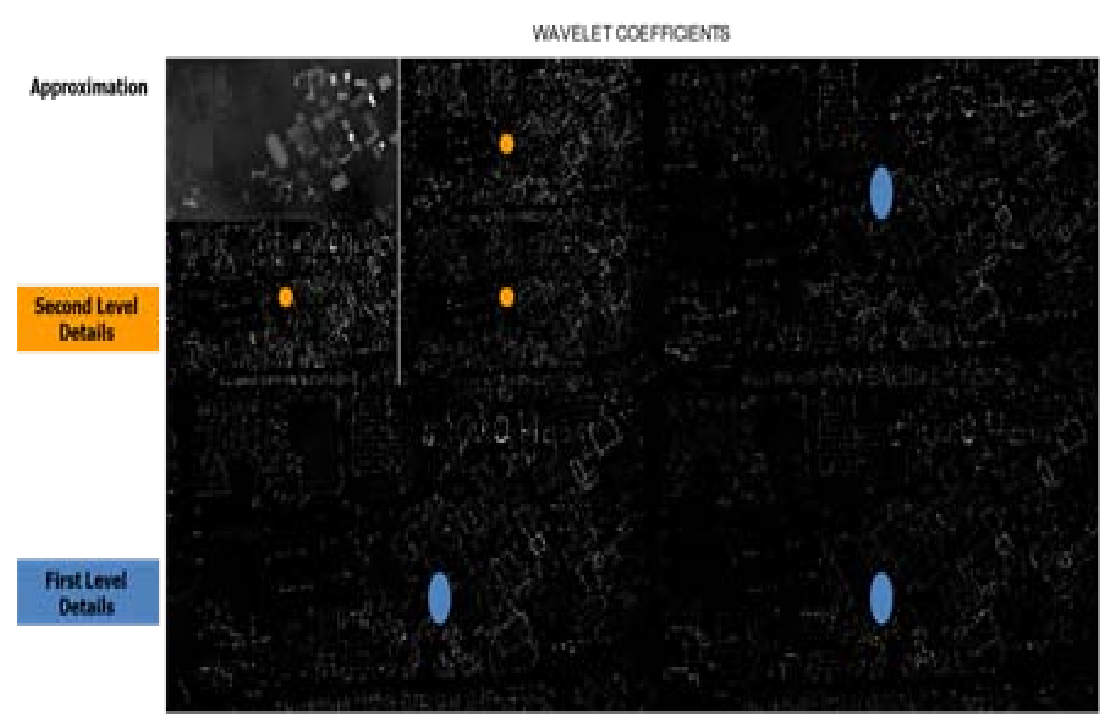

Figure 6: Wavelet Analysis for Second Level of Decomposition WAVELET COEFFICIENTS (After eliminating approximation)

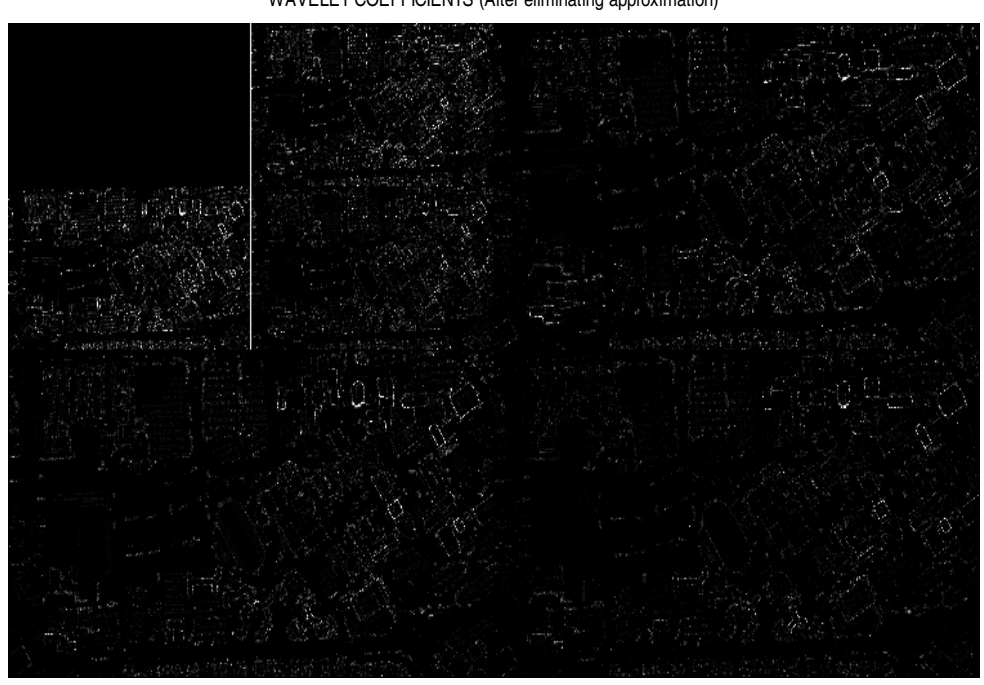


Figure 7: Wavelet detail coefficients only

\subsection{Boundary Identification}

Feature boundary identification is the important step in the building extraction algorithm since this step gives the indication of the building location. Wavelet analysis succeeded in detecting feature edges from height image because it detecting the positions of suddenly changes in height. Feature edges is detecting as high frequency components in the height image and it can be extracted after reconstruction the three details from second level of decomposition. Figure 8 shows the image of edges of all features in the study area.

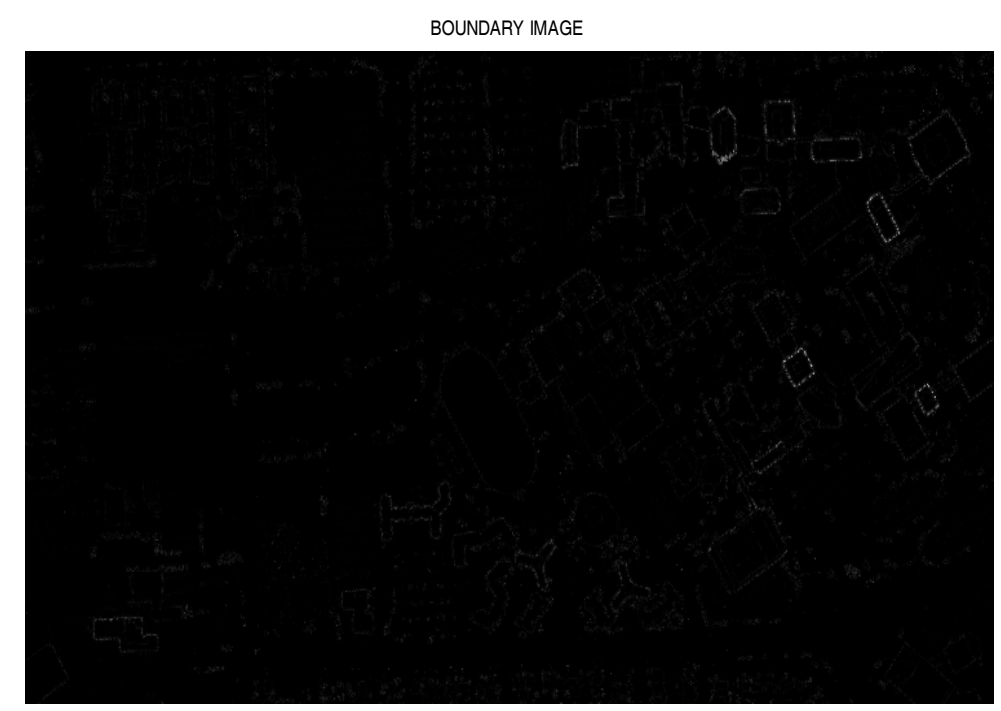

Figure 8: Boundary Image

\subsection{Production of Feature Images}

Data fusion is a process of using information from different sources to fulfil the research target. This research tends to extract features from LIDAR data, data fusion principle is used to produce building image. Building image is an image that contains only buildings without any other feature as possible. Data fusion is performed in many steps. The first step is an extraction feature image that contains all features in the image. Feature image means an image for non-ground features. Morphological filling operation is applied on boundary image to fill all extracted feature boundaries and produces a binary image (Figure 9). 
ALL FEATURES

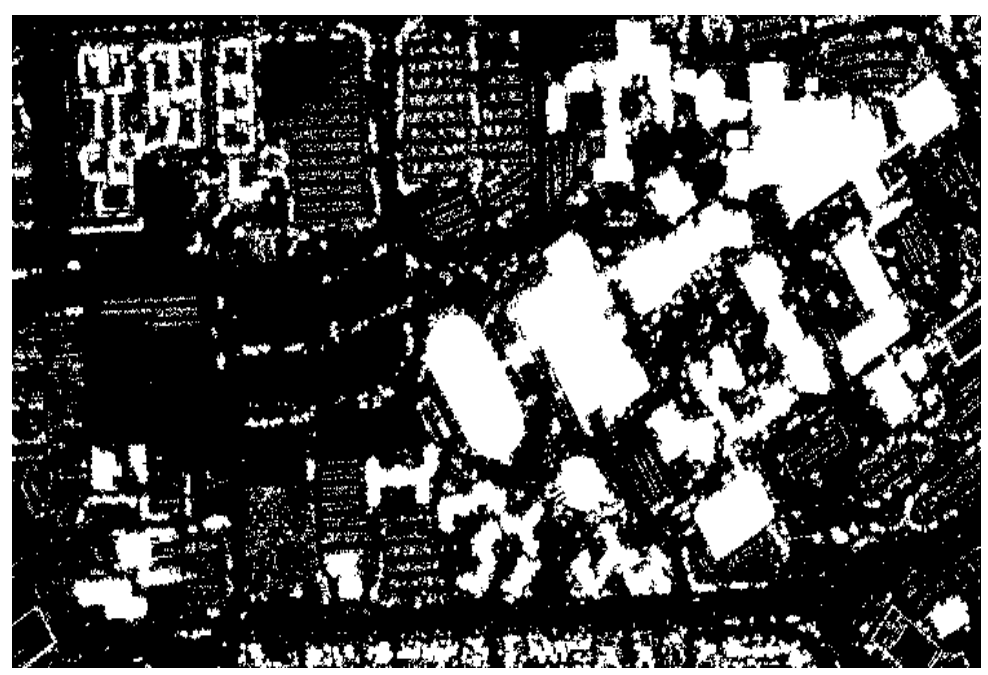

Figure 9: Feature Image

The second step is an extraction binary image for specific features that most probably represent buildings. This step is executed by applying area thresholding technique on the feature image. This technique is applies in many two steps. The first step is labelling feature image. The second step is removing labelled features that of area less than specific threshold. The resulting image is a binary buildings image (Figure 10).

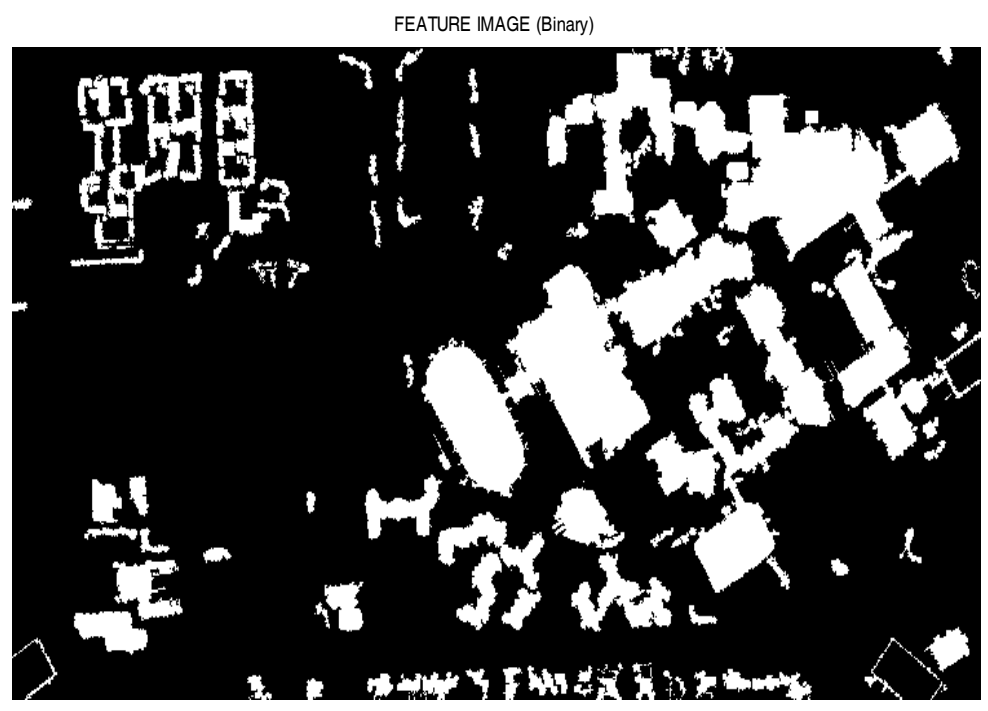

Figure 10: Binary Buildings Image

The last step of data fusion process is feeding building pixels with building information from LIDAR data. Resultant building image is based on used building information. There are many other features still appeared in the building images. These features are the vegetation areas close to the building or separate with height information nearly same or larger than buildings. Wavelet analysis succeeded in identifying feature boundaries based on identifying locations of suddenly change in height, but with no separation between features with different color or semantic information. 
LIDAR data has two different information, height and intensity, then building image can be height image (Figure 11 and Figure 12).

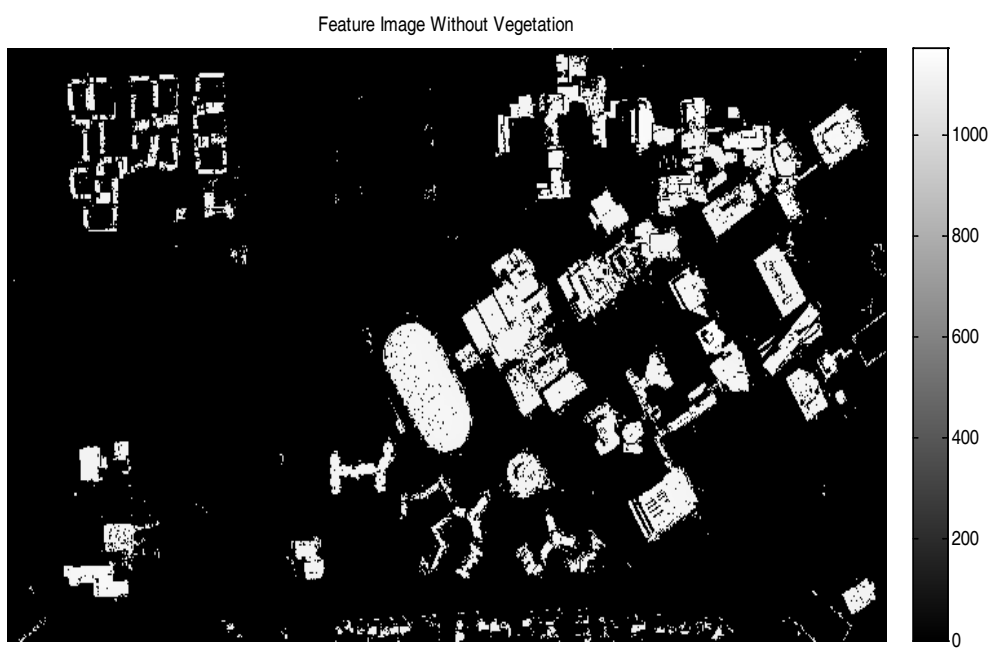

Figure 11: Height Building Image without Vegetation

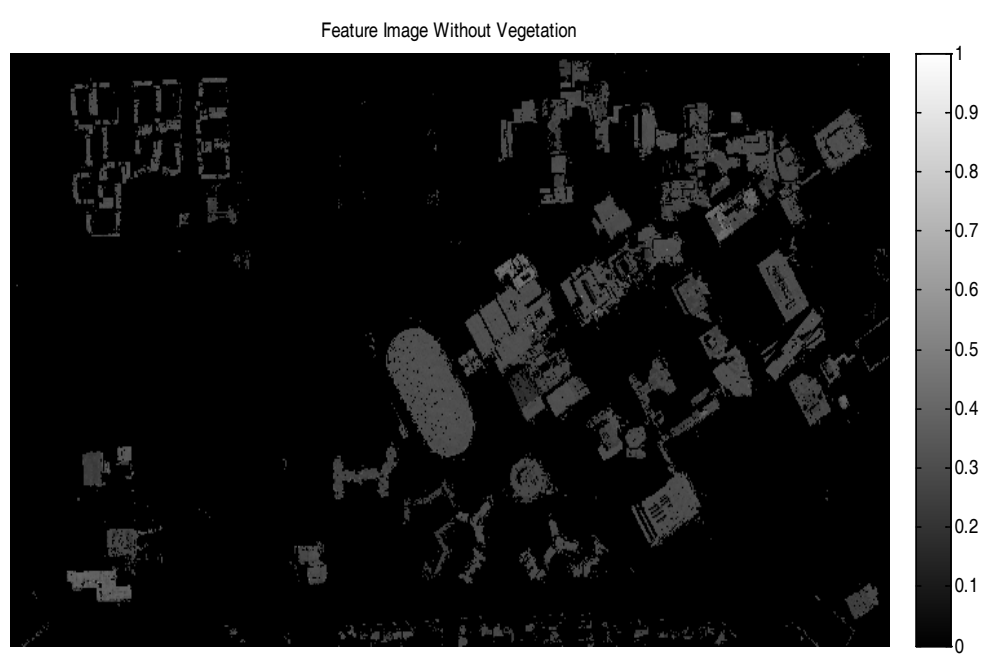

Figure 12: Intensity Building Image without Vegetation

\subsection{D Building Representation}

Ground coordinates of extracted buildings are investigated from height building image and information about ground coordinates that bounding the study area. Ground coordinates for all building pixels are investigated. The ground coordinates of other pixels with height value equal zero is replaced by approximated height value less than minimum building height. 3D building representation is obtained after applying nearest neighbour interpolation on the ground coordinates of all building pixels (Figure 13). 


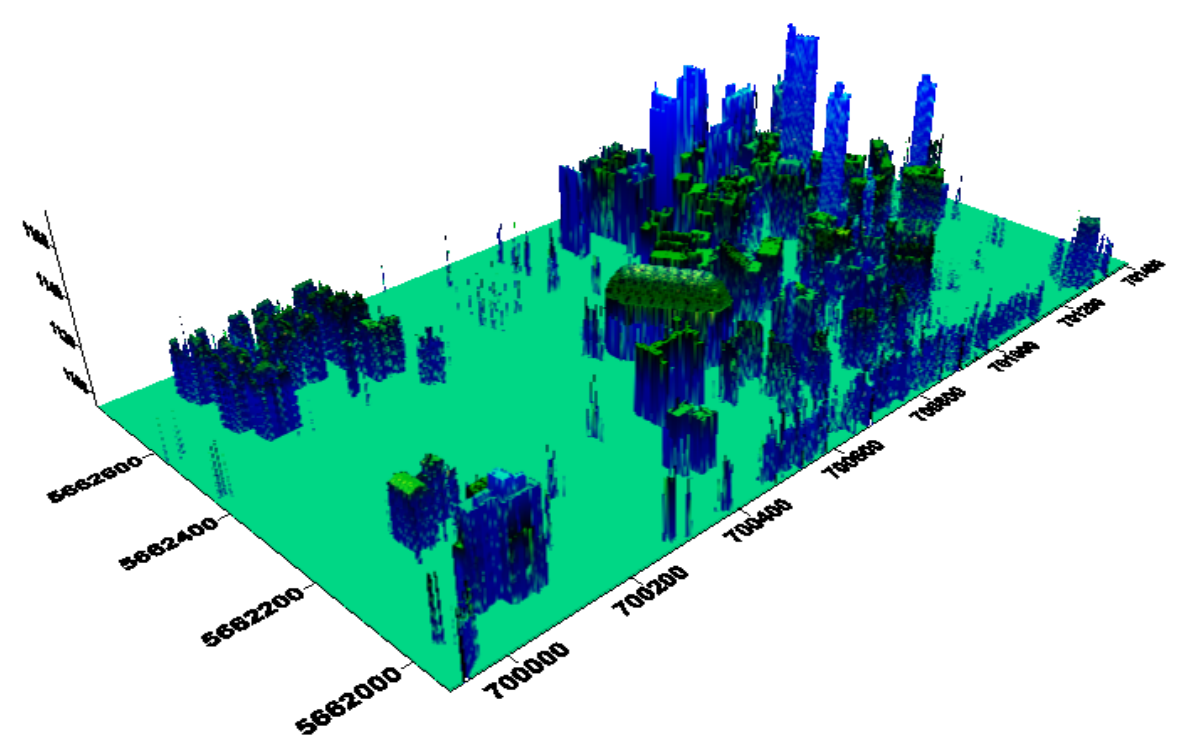

Figure 13: 3D Building Model

\section{CONCLUSIONS, AND FUTURE WORK}

LIDAR data provides dense positioning information for on terrain and off ground objects, so it produces rich spatial information for all features. Height image for an area of study which it is considered as Digital Surface Model (DSM) can be produced directly from LIDAR system. Then LIDAR system has capabilities to overcome the shortage of data from photogrammetric outputs and reduce a processing time for producing DSM for an area of study.

Wavelet is more sensitive tool for analysing signals with different frequency/space resolutions. It is a powerful analyst for identifying and localizing building boundaries from LIDAR data. This research introduced the stand alone technique based on wavelet transform for off terrain feature extraction from the LIDAR data.

For extending this research, it is recommended to search about investigation of new technique that uses results of wavelet analysis and another imaging data to provide a more powerful technique for feature extraction that will be suitable for many applications.

\section{REFERENCES}

Al-Durgham, M. (2007) Alternative methodologies for the quality control of LiDAR systems, M.Sc., University of Calgary (Canada), Canada

Bartels, M., and H. Wei (2006) "Towards DTM Generation from LIDAR Data in Hilly Terrain using Wavelets," Proceedings of 4th International Workshop on Pattern Recognition in Remote Sensing in conjunction with the 18th International Conference on Pattern Recognition, Hong Kong, China, 33-36

Cheng, H., J. W. Tian, J. Liu, and Q. Z. Yu (2004) "Wavelet domain image denoising via support vector regression," Electronics Letters, vol 40, no 23, pp. 1479-1481

El-Sheimy, N., C. Valeo, and A. Habib (2005) Digital terrain modeling : acquisition, manipulation, and applications, Artech House, Boston 
Elhabiby, M. (2007) Wavelet representation of geodetic operators, Ph.D., University of Calgary (Canada), Canada

Falkowski, M. J., A. M. S. Smith, A. T. Hudak, P. E. Gessler, L. A. Vierling, and N. L. Crookston (2006) "Automated estimation of individual conifer tree height and crown diameter via twodimensional spatial wavelet analysis of lidar data," Canadian Journal of Remote Sensing, vol 32, no 2, Apr, pp. 153-161

Gonzalez, R. C., and R. E. Woods (2002) Digital image processing, Prentice Hall, Upper Saddle River (NJ)

Gonzalez, R. C., R. E. Woods, and S. L. Eddins (2004) Digital Image processing using MATLAB, Pearson Prentice Hall, Upper Saddle River, N.J.

Habib, A., K. I. Bang, A. P. Kersting, and D. C. Lee (2009) "Error Budget of Lidar Systems and Quality Control of the Derived Data," Photogrammetric Engineering and Remote Sensing, vol 75, no 9, Sep, pp. 1093-1108

Jansen, M., and A. Bultheel (1999) "Multiple wavelet threshold estimation by generalized cross validation for images with correlated noise," IEEE Transactions on Image Processing, vol 8, no 7, pp. 947-953

Keller, W. (2004) Wavelets in geodesy and geodynamics, Walter de Gruyter, Berlin; w York

Parrish, C. E. (2008) "Exploiting full-waveform lidar data and multiresolution wavelet analysis for vertical object detection and recognition," Piscataway, NJ, USA, pp. 2499-2502, IEEE

Pradhan, B., S. Mansor, A. R. Ramli, A. R. B. Mohamed Sharif, and K. Sandeep (2005) "LIDAR data compression using wavelets," USA, pp. 598305-598301, SPIE - The International Society for Optical Engineering

Tate, N. J., C. Brunsdon, M. Charlton, A. S. Fotheringham, and C. H. Jarvis (2005) "Smoothing/filtering LiDAR digital surface models. Experiments with loess regression and discrete wavelets," Journal of Geographical Systems, vol 7, no 3-4, pp. 273-290

Vu, T. T., and M. Tokunaga (2001) "Wavelet and Scale-Space Theory in Segmentation of Airborne Laser Scanner Data " 22nd Asian Conference on Remote Sensing, 5-9 November,, Singapore

Vu, T. T., and M. Tokunaga (2002) "Wavelet-Based Clustering Method to Detect Building in Urban Area from Airborne Laser Scanner Data," In: MapAsia 2002, http://www.gisdevelopment.net/technology/ip/techip009.htm

Vu, T. T., and M. Tokunaga (2004) "Filtering airborne laser scanner data: A wavelet-based clustering method," Photogrammetric Engineering and Remote Sensing, vol 70, no 11, pp. 12671274

Wang, C.-K., and P.-H. Hsu (2006) "BUILDING EXTRACTION FROM LIDAR DATA USING WAVELET ANALYSIS," 27th Asian Conference on Remote Sensing, Ulaanbaatar, Mongolia 
Wei, H., and M. Bartels (2006) "Unsupervised segmentation using Gabor wavelets and statistical features in LIDAR data analysis," Hong Kong, China, pp. 667-670, Institute of Electrical and Electronics Engineers Inc.

Yin, S., and W. Wang (2006) "Denoising lidar signal by combining wavelet improved threshold with wavelet domain spatial filtering," Chinese Optics Letters, vol 4, no 12, pp. 694-696

Yu, T.-T., M. Yang, and C.-S. Chen (2005) "Automatic feature extraction and stereo image processing with genetic algorithms for LiDAR data," Beijing, China, pp. 307-309, Institute of Electrical and Electronics Engineers Computer Society 